\title{
Seleção de genótipos de capim-elefante quanto à resistência à cigarrinha-das-pastagens
}

\author{
Alexander Machado Auad( ${ }^{(1)}$, Amanda Daniela Simões(2), Antônio Vander Pereira ${ }^{(1)}$, André Luiz Furtado Braga ${ }^{(2)}$, \\ Fausto Souza Sobrinho(1), Francisco José da Silva Lédo(1), Silvana Viera Paula-Moraes ${ }^{(3)}$, Simone Alves Oliveira(2) \\ e Roberta Botelho Ferreira ${ }^{(2)}$
}

(1)Embrapa Gado de Leite, Rua Eugênio do Nascimento, no 610, Dom Bosco, CEP 36038-330 Juiz de Fora, MG. E-mail: amauad@cnpgl.embrapa.br, avanderp@cnpgl.embrapa.br, fausto@cnpgl.embrapa.br, ledo@cnpgl.embrapa.br ${ }^{(2)}$ Centro de Ensino Superior de Juiz de Fora, Av. Luz Interior, no 345, Estrela Sul, CEP 36016-000 Juiz de Fora, MG. E-mail: amanda-daniela@ig.com.br, alfbraga@gmail.com, sibio@yahoo.com.br, robertabotelhoferreira@yahoo.com.br (3)Embrapa Cerrados, BR 020, Km 18, CEP 73310-970 Planaltina, DF. E-mail: silvana@cpac.embrapa.br

Resumo - O objetivo deste trabalho foi avaliar genótipos de capim-elefante (Pennisetum purpureum Schum.) quanto à resistência à cigarrinha-das-pastagens (Mahanarva spectabilis). Para avaliação da antibiose, aos trinta dias após o plantio, cada planta foi infestada com seis ovos próximos à eclosão, em delineamento inteiramente casualizado, com 30 genótipos e dez repetições. Quarenta e cinco dias após a eclosão das ninfas, avaliou-se a porcentagem de sobrevivência do inseto-praga nos diferentes genótipos. Para avaliação da não-preferência, foram quantificados, quinzenalmente, o número e tamanho de ninfas por vaso, em plantas mantidas em casa de vegetação, onde adultos de $M$. spectabilis eram periodicamente liberados, em delineamento inteiramente casualizado, com três repetições por genótipo, em dois períodos de amostragem. Os genótipos Cameroon de Piracicaba, Pioneiro, Cuba 169, Santa Rita, Mineiro Ipeaco, Mercker Comum de Pinda e CNPGL 96-27-3 foram selecionados quanto à resistência, pelo mecanismo de antibiose. O número e o tamanho médio das ninfas variaram significativamente em razão do genótipo de capim-elefante, no estudo da não-preferência. Os genótipos Roxo de Botucatu e Pioneiro são candidatos à testemunha suscetível e resistente, respectivamente, pelo mecanismo de antibiose, e os genótipos Cameroon e Cameroon Piracicaba são promissores pelo mecanismo de não-preferência.

Termos para indexação: Pennisetum purpureum, Mahanarva spectabilis, resistência a pragas, forrageiras, antibiose, não-preferência.

\section{Selection of elephant grass genotypes for resistance to spittlebug}

\begin{abstract}
The objective of this work was to evaluate genotypes of elephant grass (Pennisetum purpureum Schum.) regarding resistance to the spittlebug Mahanarva spectabilis. For antibiosis evaluation, each plant was infested with six eggs near hatching, thirty days after planting, in a completely randomized design with 30 genotypes and ten repetitions. Forty-five days after the nymphs hatched, the insect survival rate was evaluated on the different genotypes. For nonpreference mechanism evaluation, the size and number of the nymphs per pot were assessed every 15 days, in plants kept at greenhouse, where adults of $M$. spectabilis were periodically released, in a completely randomized design, with three repetitions per genotype in two sampling periods. Cameroon de Piracicaba, Pioneiro, Cuba 169, Santa Rita, Mineiro Ipeaco, Mercker Comum de Pinda and CNPGL 96-27-3 genotypes were selected for resistance, by antibiosis mechanism. In the study of the nonpreference mechanism, the number and average size of the nymphs varied significantly as a function of the elephant grass genotype. Roxo de Botucatu and Pioneiro genotypes are, respectively, susceptible and resistant check candidates by the antibiosis mechanism, and Cameroon and Cameroon Piracicaba genotypes are promising by the nonpreference mechanism.
\end{abstract}

Index terms: Pennisetum purpureum, Mahanarva spectabilis, pest resistance, forage, antibiosis, nonpreference.

\section{Introdução}

O capim-elefante (Pennisetum purpureum Schum.) é uma das forrageiras que mais tem contribuído para a alimentação animal em sistemas de produção de leite.
Além da sua comprovada superioridade para formação de capineiras, Martins et al. (1993) e Deresz \& Mozzer (1997) demonstraram que essa forrageira apresenta um excelente comportamento para uso sob pastejo rotativo. Todavia, a ocorrência da cigarrinha-das-pastagens 
Mahanarva spectabilis (Distant, 1909) (Hemiptera: Cercopidae) pode limitar o cultivo dessa gramínea.

Essa cigarrinha-das-pastagens é considerada praga limitante na produção de gramíneas forrageiras. A redução na capacidade de suporte das pastagens, imposta pela ação das cigarrinhas, é problema relevante na bovinocultura brasileira (Valério, 2005). Apesar de existir um grande número de referências sobre a resistência de várias forrageiras à cigarrinha-daspastagens (Lapointe et al., 1992; Valério et al., 1997; Figueiredo et al., 2001; Cardona et al., 2004), as relativas ao capim-elefante são raras.

Uma das principais demandas dos produtores de leite, em relação às instituições de pesquisas, refere-se à obtenção de cultivares de capim-elefante resistentes às cigarrinhas. Contudo, pesquisas que tratam mais diretamente do relacionamento desse inseto com a forrageira são incomuns. A ausência dessas informações tem limitado as recomendações de medidas de controle que poderiam contribuir para a minimização dos impactos causados às capineiras.

O objetivo deste trabalho foi avaliar genótipos de capim-elefante quanto à resistência a M. spectabilis.

\section{Material e Métodos}

A criação de $M$. spectabilis da Embrapa Gado de Leite, em Juiz de Fora, MG, serviu de suprimento de material vivo para os ensaios que foram realizados em casa de vegetação (temperatura mínima, média e máxima de 20,29 e $36^{\circ} \mathrm{C}$, respectivamente).

Adultos do inseto-praga foram levados para o laboratório, sexados e mantidos em gaiolas cilíndricas de plástico transparente $(50 \mathrm{~cm}$ de altura $\mathrm{x} 10 \mathrm{~cm}$ de diâmetro). Em cada gaiola foi colocada uma planta de capim-elefante com a base envolvida por gaze umedecida em água destilada, que serviu de substrato para oviposição. Para a retirada dos ovos retidos no substrato, a gaze foi colocada sobre um conjunto de peneiras e submetido à água corrente, tendo os ovos ficado retidos naquela mais fina (400 mesh de abertura). Posteriormente, os ovos obtidos foram colocados em placas de Petri de $5 \mathrm{~cm}$ de diâmetro, forradas com papelfiltro e mantidas em câmara climatizadas $\left(28 \pm 2^{\circ} \mathrm{C}\right.$, 14 horas de fotófase e umidade relativa de $70 \pm 10 \%$ ) até próximo à eclosão.

Para o estudo da antibiose, analisaram-se 30 genótipos de capim-elefante (Tabela 1). Estabeleceram-se dez plantas de cada genótipo, em vasos de $500 \mathrm{~mL}$, a partir de estacas de um nó. Após 30 dias do plantio das estacas, cada planta foi infestada com seis ovos, previamente incubados em laboratório e próximos à eclosão.

Quarenta e cinco dias após a eclosão, foi realizada a contagem das ninfas de primeiro, segundo, terceiro, quarto e quinto ínstares, bem como do número total de ninfas sobreviventes, nos diferentes genótipos de capim-elefante.

$\mathrm{O}$ delineamento experimental foi inteiramente casualizado, com dez repetições. Os dados foram transformados em $(\mathrm{x}+1)^{0,5}$ e submetidos à análise de variância, com as médias comparadas pelo teste de ScottKnott, a 5\% de probabilidade. Realizou-se também a análise que considerava a média geral do ensaio e o desvio-padrão experimental.

Tabela 1. Sobrevivência média de ninfas de Mahanarva spectabilis de terceiro, quarto e quinto ínstares (N3+N4+N5) e sobrevivência total $(\mathrm{N} 1+\mathrm{N} 2+\mathrm{N} 3+\mathrm{N} 4+\mathrm{N} 5)$, em diferentes genótipos de capim-elefante ${ }^{(1)}$.

\begin{tabular}{lcr}
\hline Genótipo & \multicolumn{2}{c}{ Sobrevivência (\%) } \\
\cline { 2 - 3 } & N3+N4+N5 & Total \\
\hline Cameroon Piracicaba & $35,42 \mathrm{~b}$ & $37,50 \mathrm{~b}$ \\
Pioneiro & $33,33 \mathrm{~b}$ & $41,67 \mathrm{~b}$ \\
Cuba 169 & $40,00 \mathrm{~b}$ & $43,33 \mathrm{~b}$ \\
Santa Rita & $38,10 \mathrm{~b}$ & $47,62 \mathrm{~b}$ \\
Mineiro Ipeaco & $33,33 \mathrm{~b}$ & $47,62 \mathrm{~b}$ \\
Elefante da Colômbia & $54,17 \mathrm{a}$ & $58,33 \mathrm{~b}$ \\
Mecker Comum de Pinda & $50,00 \mathrm{~b}$ & $59,52 \mathrm{~b}$ \\
CNPGL 96-27-3 & $41,67 \mathrm{~b}$ & $60,42 \mathrm{~b}$ \\
P241 Piracicaba & $56,25 \mathrm{a}$ & $64,58 \mathrm{a}$ \\
Mercker Comum & $57,14 \mathrm{a}$ & $64,58 \mathrm{a}$ \\
Mercker x 23A (híbrido triplóide) & $56,25 \mathrm{a}$ & $64,58 \mathrm{a}$ \\
Napier & $58,33 \mathrm{a}$ & $66,67 \mathrm{a}$ \\
Taiwan A-146 & $64,58 \mathrm{a}$ & $66,67 \mathrm{a}$ \\
Capim Cana d'África & $60,42 \mathrm{a}$ & $66,67 \mathrm{a}$ \\
Napier x 23 A (híbrido triplóide) & $60,42 \mathrm{a}$ & $68,75 \mathrm{a}$ \\
Taiwan A-144 & $64,29 \mathrm{a}$ & $69,05 \mathrm{a}$ \\
Vrukwona & $57,14 \mathrm{a}$ & $69,05 \mathrm{a}$ \\
Cameroon & $66,67 \mathrm{a}$ & $70,83 \mathrm{a}$ \\
IJ 7136 cv. Empasc 307 & $50,00 \mathrm{~b}$ & $71,43 \mathrm{a}$ \\
Porto Rico & $60,42 \mathrm{a}$ & $72,92 \mathrm{a}$ \\
Guaçu I.Z. 2 & $60,42 \mathrm{a}$ & $77,08 \mathrm{a}$ \\
Mott & $76,19 \mathrm{a}$ & $78,57 \mathrm{a}$ \\
IJ 7141 cv. Empasc 306 & $66,67 \mathrm{a}$ & $79,17 \mathrm{a}$ \\
Sem Pêlo & $76,19 \mathrm{a}$ & $80,95 \mathrm{a}$ \\
Kizozi & $68,75 \mathrm{a}$ & $81,25 \mathrm{a}$ \\
King Grass & $71,66 \mathrm{a}$ & $81,67 \mathrm{a}$ \\
CNPGL 94-49-6 & $72,92 \mathrm{a}$ & $85,41 \mathrm{a}$ \\
CNPGL 94-43-1 & $61,11 \mathrm{a}$ & $86,11 \mathrm{a}$ \\
Mineiro & $72,22 \mathrm{a}$ & $88,89 \mathrm{a}$ \\
Roxo de Botucatu & $91,66 \mathrm{a}$ & $100,00 \mathrm{a}$ \\
\hline
\end{tabular}

${ }^{(1)}$ Médias seguidas por letras iguais, nas colunas, não diferem entre si pelo teste de Scott-Knott, a 5\% de probabilidade. 
A avaliação do mecanismo de resistência por nãopreferência foi realizada nos genótipos de capim-elefante mantido em vasos, na casa de vegetação da Embrapa Gado de Leite, onde as plantas eram mantidas à temperatura oscilante e irrigadas por aspersão diariamente; isso criou um ambiente favorável para criação de $M$. spectabilis, coletadas no campo experimental e liberadas periodicamente na casa de vegetação. Quinzenalmente, foram registrados o número e o tamanho de ninfas do inseto-praga por vaso, de setembro de 2005 a abril de 2006, em 23 genótipos e, de agosto de 2006 a março de 2007, com intuito de ampliar o número de genótipos a serem testados, foram utilizados 30 genótipos de capim-elefante. As ninfas foram agrupadas de acordo com o ínstar, tendo sido consideradas pequenas as de primeiro e segundo ínstares; médias, as de terceiro e quarto ínstares; e grandes, as de quinto ínstar (Tabela 2). O delineamento experimental foi inteiramente casualizado, com três repetições por genótipo, no total de 69 e 90 vasos, no primeiro e no segundo períodos de amostragem, respectivamente. Os dados foram transformados em $(\mathrm{x}+1)^{0,5} \mathrm{e}$ submetidos à análise de variância, com as médias comparadas pelo teste de Scott-Knott, a 5\% de probabilidade.

\section{Resultados e Discussão}

Houve efeito significativo dos genótipos, na sobrevivência média de ninfas de $M$. spectabilis de terceiro a quinto ínstar e total ( $p<0,01)$, o que evidencia a existência de variabilidade entre os genótipos de capim-elefante quanto à resistência à cigarrinha-das-pastagens, pelo mecanismo de antibiose. A sobrevivência média de ninfas de primeiro a quinto ínstar de M. spectabilis, nos trinta genótipos de capim-elefante, variou entre 37,5 e $100 \%$ (Tabela 1).

Tabela 2. Número de ninfas de diferentes tamanhos de Mahanarva spectabilis, coletadas nos genótipos de capim-elefante, em dois períodos de amostragem ${ }^{(1)}$.

\begin{tabular}{|c|c|c|c|c|c|c|c|c|}
\hline \multirow[t]{2}{*}{ Genótipo } & \multicolumn{4}{|c|}{$2005 / 2006$} & \multicolumn{4}{|c|}{$2006 / 2007$} \\
\hline & Pequena & Média & Grande & Total & Pequena & Média & Grande & Total \\
\hline Vrukwona & $1,13 \mathrm{a}$ & $1,47 \mathrm{a}$ & $0,50 \mathrm{~b}$ & $3,09 b$ & 5,39a & $0,68 \mathrm{a}$ & $0,32 \mathrm{a}$ & $6,39 a$ \\
\hline Sem Pêlo & $1,50 \mathrm{a}$ & $1,34 \mathrm{a}$ & $0,47 b$ & $3,31 b$ & $4,55 \mathrm{a}$ & $0,76 \mathrm{a}$ & $0,12 b$ & $5,42 \mathrm{a}$ \\
\hline Pioneiro & $1,04 b$ & $1,08 \mathrm{~b}$ & $0,20 \mathrm{c}$ & $2,31 \mathrm{c}$ & $1,33 b$ & $2,62 \mathrm{a}$ & $0,48 \mathrm{a}$ & $4,42 \mathrm{a}$ \\
\hline CNPGL 96-27-3 & $0,84 b$ & $1,41 \mathrm{a}$ & $0,53 b$ & $2,78 \mathrm{c}$ & $3,45 \mathrm{a}$ & $0,79 a$ & $0,03 b$ & $4,30 \mathrm{a}$ \\
\hline Elefante da Colômbia & $0,59 b$ & $0,88 \mathrm{~b}$ & $0,13 c$ & $1,59 \mathrm{~d}$ & $3,12 \mathrm{a}$ & $1,03 \mathrm{a}$ & $0,06 \mathrm{~b}$ & $4,21 \mathrm{a}$ \\
\hline Santa Rita & $1,38 \mathrm{a}$ & $1,63 a$ & $0,41 b$ & $3,41 b$ & $3,05 \mathrm{a}$ & $0,67 \mathrm{a}$ & $0,05 b$ & $3,77 \mathrm{a}$ \\
\hline Kizozi & $0,34 b$ & $0,25 b$ & $0,00 \mathrm{c}$ & $0,59 \mathrm{~d}$ & $1,50 \mathrm{~b}$ & $2,13 \mathrm{a}$ & $0,00 \mathrm{~b}$ & $3,63 \mathrm{a}$ \\
\hline Guaçu I.Z. 2 & $0,84 b$ & $1,84 \mathrm{a}$ & $0,63 b$ & $3,31 b$ & $2,06 \mathrm{~b}$ & $1,20 \mathrm{a}$ & $0,35 \mathrm{a}$ & $3,59 a$ \\
\hline Cuba 169 & $1,16 \mathrm{a}$ & $1,84 \mathrm{a}$ & $0,69 b$ & $3,69 b$ & $1,67 b$ & $1,60 \mathrm{a}$ & $0,30 \mathrm{a}$ & $3,57 \mathrm{a}$ \\
\hline Mott & $1,38 \mathrm{a}$ & $1,50 \mathrm{a}$ & $0,63 b$ & $3,50 \mathrm{~b}$ & $2,14 b$ & $0,96 \mathrm{a}$ & $0,67 \mathrm{a}$ & $3,45 \mathrm{a}$ \\
\hline Mineiro & $0,61 b$ & $1,06 b$ & $0,28 \mathrm{c}$ & $1,95 \mathrm{~d}$ & $2,02 b$ & $1,24 \mathrm{a}$ & $0,10 \mathrm{~b}$ & $3,36 \mathrm{a}$ \\
\hline Mineiro Ipeaco & $1,19 \mathrm{a}$ & $1,38 \mathrm{a}$ & $0,16 \mathrm{c}$ & $2,72 \mathrm{c}$ & $1,93 b$ & $1,13 \mathrm{a}$ & $0,25 b$ & $3,31 \mathrm{a}$ \\
\hline IJ 7136 cv. Empasc 307 & $2,09 \mathrm{a}$ & $2,19 \mathrm{a}$ & $0,50 \mathrm{~b}$ & $4,78 \mathrm{a}$ & $1,89 b$ & $0,73 \mathrm{a}$ & $0,15 b$ & $2,76 \mathrm{~b}$ \\
\hline Napier & $1,10 \mathrm{a}$ & $1,10 \mathrm{~b}$ & $0,17 \mathrm{c}$ & $2,38 \mathrm{c}$ & $1,43 b$ & $0,80 \mathrm{a}$ & $0,43 \mathrm{a}$ & $2,66 b$ \\
\hline Roxo de Botucatu & $1,50 \mathrm{a}$ & $2,16 a$ & $0,41 b$ & $4,06 a$ & $1,73 b$ & $0,67 \mathrm{a}$ & $0,18 b$ & $2,58 \mathrm{~b}$ \\
\hline Cameroon & $0,56 b$ & $0,78 b$ & $0,34 \mathrm{c}$ & $1,69 d$ & $2,11 b$ & $0,28 \mathrm{a}$ & $0,06 b$ & $2,44 b$ \\
\hline IJ 7141 cv. Empasc 306 & $1,44 \mathrm{a}$ & $1,69 \mathrm{a}$ & $1,06 \mathrm{a}$ & $4,19 \mathrm{a}$ & $1,72 b$ & $0,41 \mathrm{a}$ & $0,13 b$ & $2,26 \mathrm{~b}$ \\
\hline Cameroon Piracicaba & $0,59 b$ & $0,88 b$ & $0,29 c$ & $1,76 \mathrm{~d}$ & $0,83 b$ & $1,11 \mathrm{a}$ & $0,28 \mathrm{a}$ & $2,22 b$ \\
\hline King Grass & $0,72 b$ & $1,44 \mathrm{a}$ & $0,41 b$ & $2,56 \mathrm{c}$ & $1,17 b$ & $0,66 \mathrm{a}$ & $0,07 \mathrm{~b}$ & $1,90 \mathrm{~b}$ \\
\hline Porto Rico & $0,81 b$ & $1,47 \mathrm{a}$ & $0,16 \mathrm{c}$ & $2,44 \mathrm{c}$ & $0,15 b$ & $0,96 \mathrm{a}$ & $0,00 \mathrm{~b}$ & $1,12 b$ \\
\hline P241 Piracicaba & $1,47 \mathrm{a}$ & $0,66 b$ & $0,31 \mathrm{c}$ & $2,44 \mathrm{c}$ & $0,67 b$ & $0,28 \mathrm{a}$ & $0,00 \mathrm{~b}$ & $0,94 b$ \\
\hline Mercker Comum de Pinda & -- & -- & -- & -- & $3,00 \mathrm{a}$ & $1,45 \mathrm{a}$ & $0,18 b$ & $4,64 \mathrm{a}$ \\
\hline Mercker x 23A (h. triplóide) & -- & -- & -- & -- & $2,57 \mathrm{~b}$ & $1,20 \mathrm{a}$ & $0,33 \mathrm{a}$ & $4,10 \mathrm{a}$ \\
\hline CNPGL 94-49-6 & -- & -- & -- & -- & $1,96 b$ & $1,52 \mathrm{a}$ & $0,57 \mathrm{a}$ & $4,06 \mathrm{a}$ \\
\hline Napier x 23A (h. triplóide) & -- & -- & -- & -- & $2,00 \mathrm{~b}$ & $0,28 \mathrm{a}$ & $0,00 \mathrm{~b}$ & $2,28 b$ \\
\hline CNPGL 94-43-1 & -- & -- & -- & -- & $1,38 b$ & $0,81 \mathrm{a}$ & $0,06 \mathrm{~b}$ & $2,25 b$ \\
\hline Taiwan A -146 & -- & -- & -- & -- & $0,66 b$ & $0,71 \mathrm{a}$ & $0,15 b$ & $1,51 \mathrm{~b}$ \\
\hline Mercker Comum & -- & -- & -- & -- & $0,87 \mathrm{~b}$ & $0,22 \mathrm{a}$ & $0,20 \mathrm{~b}$ & $1,29 b$ \\
\hline
\end{tabular}

${ }^{(1)}$ Médias seguidas por letras iguais, nas colunas, não diferem entre si pelo teste de Scott-Knott, a 5\% de probabilidade. 
As menores médias de porcentagens de sobrevivência da cigarrinha foram verificadas nos genótipos Cameroon de Piracicaba, Pioneiro, Cuba 169, Santa Rita, Mineiro Ipeaco, Elefante da Colômbia, Mercker Comum de Pinda e CNPGL 96-27-3 (Tabela 1). Para os demais genótipos, constatou-se sobrevivência, no número total de ninfas, acima de $64 \%$, o que é evidência de suscetibilidade. Quando a análise dos dados foi realizada, considerandose apenas a porcentagem de sobrevivência de ninfas de terceiro a quinto ínstar, verificou-se variação de 33,3 a 91,7\% (Tabela 1). A antibiose como mecanismo de resistência de braquiárias à cigarrinha-das-pastagens foi registrada por Lapointe et al. (1992), Valério et al. (1997), Cardona et al. (2004) e Miles et al. (2006). No entanto, para capim-elefante, as pesquisas ainda são incipientes, $\mathrm{o}$ que dificulta a recomendação de genótipos para regiões com histórico de problema com esse inseto-praga.

A diferença entre porcentagem de sobrevivência total e de ninfas de terceiro a quinto ínstar foi mais evidente no genótipo IJ 7136 cultivar Empasc 307; considerando-se a sobrevivência média total ou daquelas de terceiro a quinto ínstar, esse genótipo se comportou como material suscetível ou resistente à M. spectabilis, respectivamente (Tabela 1). Assim, adotou-se como critério para a identificação e seleção dos genótipos resistentes de capim-elefante à $M$. spectabilis, a porcentagem de ninfas sobreviventes de terceiro a quinto ínstar, tendo-se excluído as de primeiro e segundo ínstares. Esse critério para seleção de resistência por antibiose, apoiado pelo fato de que ninfas com 45 dias de idade ainda não atingiram o terceiro ínstar, indica que a planta está promovendo condição desfavorável ao desenvolvimento do inseto-praga.

Os clones Pioneiro, Cameroon Piracicaba, Mineiro Ipeaco, Santa Rita e Cuba 169, em que o grau de sobrevivência foi abaixo da média do grupo menos o valor do desvio-padrão correspondente, foram considerados resistentes, provavelmente com antibiose como mecanismo de resistência. A expressão de antibiose foi também registrada por Cardona et al. (2004), que relataram alta mortalidade de Mahanarva trifissa em braquiárias resistentes. Os genótipos Napier, Vrukwona, Mercker Comum, Mercker x 23A, P241 Piracicaba, Elefante da Colômbia, IJ 7136 cultivares Empasc 307, Mercker Comum de Pinda e CNPGL 96-27-3 tiveram comportamento similar, porém, apresentaram as médias de sobrevivência entre a média geral e o desvio-padrão. Nos demais materiais, a sobrevivência média foi superior à média geral somada ao desvio-padrão, o que indica serem materiais de maior suscetibilidade à M. spectabilis.
No ensaio do mecanismo de resistência por nãopreferência, verificou-se que o número de ninfas pequenas (primeiro e segundo ínstares), médias (terceiro e quarto ínstares), grandes (quinto ínstar) e totais, nos genótipos avaliados, foi significativamente diferente $(\mathrm{p}<0,01)$, em ambos os períodos de amostragem (Tabela 2).

Nas avaliações de setembro de 2005 a abril de 2006 e de agosto de 2006 a março de 2007 (Tabela 2), foi constatado que o número médio de ninfas de primeiro e segundo ínstares foi significativamente diferente, nos genótipos avaliados quanto ao mecanismo de nãopreferência, o que evidencia haver algum fator regulador da população desse inseto. Essa oscilação do número de ninfas de primeiro e segundo ínstares de $M$. spectabilis, nos diferentes genótipos, pode ser explicada pelo mecanismo de não-preferência dos adultos para oviposição. Pode-se ainda supor que mesmo os ovos tendo sido depositados, as ninfas emergidas não sobreviveram, por fatores deletérios da planta.

Para os ínstares subseqüentes, constatou-se, também, uma oscilação no número de ninfas amostradas, em função do genótipo avaliado, o que determina que alguns materiais possuem fatores que afetam a sobrevivência ninfal, conforme já detectado no ensaio anterior.

$\mathrm{Na}$ avaliação do número de ninfas pequenas, médias, grandes e total de M. spectabilis, em 2005/2006, constatouse que os genótipos Mineiro, Cameroon Piracicaba, Cameroon, Elefante da Colômbia e Kizozi foram os que apresentaram o menor número de ninfas, o que torna esses materiais promissores, visto que além de menos preferidos pelas cigarrinhas (Tabela 2), foram também os materiais que promoveram menor sobrevivência no ensaio de antibiose, exceto Cameroon e Kizozi (Tabela 1). O número total de ninfas foi superior nos genótipos IJ 7136 cv. Empasc 307, Taiwan A-144, IJ 7141 cv. Empasc 306, Roxo de Botucatu (Tabela 2), que também apresentaram maior sobrevivência no ensaio de antibiose (Tabela 1), e valores intermediários foram verificados nos demais genótipos (Tabela 2). Esses resultados evidenciam a possibilidade não só de identificação e seleção de materiais de capim-elefante resistentes à cigarrinha-das-pastagens, mas também de alguns genótipos que associam antibiose e não-preferência como mecanismos de resistência.

De agosto de 2006 a março de 2007, o número total de ninfas foi classificado em dois grupos pelo teste de ScottKnott, que apresentaram porcentagens médias de sobrevivência de 3,31 a 6,39 ninfas por vaso e 0,94 a 2,76 ninfas por vaso. 
A densidade populacional de ninfas de $M$. spectabilis pequenas, médias e grandes, nas diferentes épocas de amostragens, está apresentada na Figura 1. Em 2005/2006, os picos populacionais de ninfas foram constatados nos meses de setembro (303 ninfas), na primeira quinzena de dezembro (272 ninfas), nas duas amostragens do mês de março (232 e 299 ninfas) e em abril (392 ninfas). No período de 2006/2007, foi constatada a maior densidade populacional de ninfas na segunda quinzena de novembro (829 ninfas), na primeira de dezembro (969 ninfas) e na primeira de janeiro (638 ninfas). Nessas ocasiões, a temperatura média foi de $26^{\circ} \mathrm{C}$, que é favorável para a ocorrência das maiores densidades populacionais desse inseto-praga.
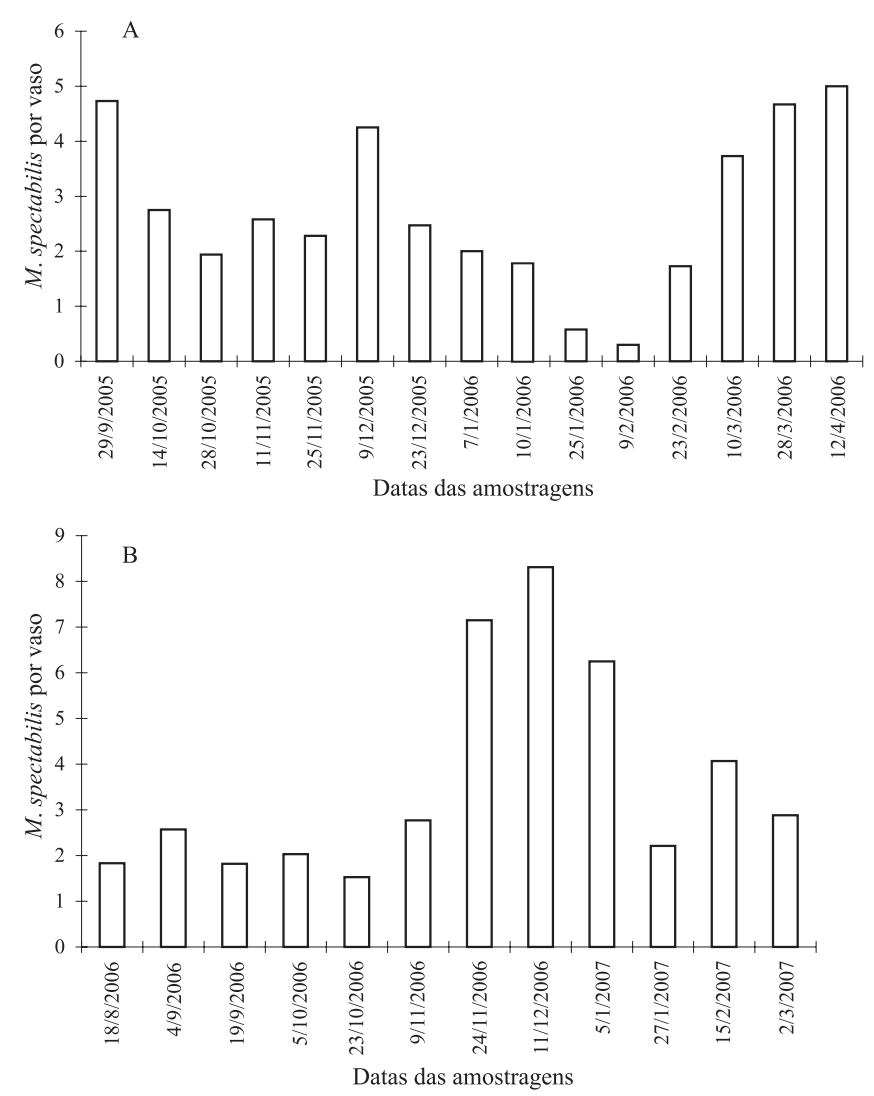

Figura 1. Número de ninfas de Mahanarva spectabilis, amostradas de setembro de 2005 a abril de 2006 (A) e agosto de 2006 a março de 2007 (B), nos diferentes genótipos de capim-elefante.

\section{Conclusão}

1. Os genótipos de capim-elefante apresentam diferenças quanto à resistência à Mahanarva spectabilis.

2. Os genótipos Roxo de Botucatu e Pioneiro são, respectivamente, candidatos a testemunhas suscetível e resistente, pelo mecanismo de antibiose; Cameroon e Cameroon Piracicaba são os mais promissores como resistentes, pelo mecanismo de não-preferência.

\section{Agradecimentos}

Ao Dr. Gervásio Silva Carvalho, da Pontifícia Universidade Católica do Rio Grande do Sul, pela identificação da espécie de cigarrinha.

\section{Referências}

CARDONA, C.; FORY, P.; SOTELO, G.; PABON, A.; DIAZ, G.; MILES, J.W. Antibiosis and tolerance to five species of spittlebug (Homoptera: Cercopidae) in Brachiaria spp.: implications for breeding for resistance. Journal of Economic Entomology, v.97, p.635-645, 2004.

DERESZ, F.; MOZZER, O.L. Produção de leite em pastagem de capim-elefante. In: CARVALHO, M.M.; ALVIM, M.J.; XAVIER, D.F. (Org.). Capim-elefante: produção e utilização. 2.ed. Brasília: Embrapa-SPI, 1997. p.155-172.

FIGUEIREDO, D.M.; VALÉRIO, J.R.; BARBOSA, I.R.; CONTE, C.O.; OLIVEIRA, M.C.M. 2001. Avaliação de danos causados por adultos da cigarrinha-das-pastagens Deois flavopicta (Homoptera: Cercopidae) em introduções e cultivares de Panicum maximum. In: SCHENK, M.A.M.; LIMA, E.C.N.Z.; CINTRA, M.AM. de U.; COSTA, F.P. Despertando vocações: a Embrapa Gado de Corte pesquisando com estudante. Campo Grande: Embrapa Gado de Corte, 2001. p.19-20. (Embrapa Gado de Corte. Documentos, 107).

LAPOINTE, S.L.; SERRANO, M.S.; ARANGO, G.L.; SOTELO, G.; CORDOBA, F. Antibiosis to spittlebugs (Homoptera: Cercopidae) in accessions of Brachiaria spp. Journal of Economic Entomology, v.85, p.1485-1490, 1992.

MARTINS, C.E.; DERESZ, F.; MATOS, L.L. Produção intensiva de leite em pasto de capim-elefante. Informações Agronômicas, v.62, p.1-4, 1993.

MILES, J.W.; CARDONA, C.; SOTELO, G. Recurrent selection in a synthetic brachiaria grass population improves resistance to three spittlebug species. Crop Science, v.46, p.1088-1093, 2006.

VALÉRIO, J.R. Insetos-praga em pastagens tropicais. Informe Agropecuário, v.26, p.98-110, 2005.

VALÉRIO, J.R.; JELLER, H.; PEIXER, J. Seleção de introduções do gênero Brachiaria (Griseb) resistentes à cigarrinha Zulia entreriana (Berg) (Homoptera: Cercopidae). In: Anais da Sociedade Entomológica do Brasil, v.26, p.383-387, 1997. 